\title{
ERGOTROPY OF BOSONIC QUANTUM BATTERY DRIVEN VIA REPELLING FEEDBACK ALGORITHMS
}

\author{
Sergey Borisenok \\ Department of Electrical and Electronics Engineering \\ Faculty of Engineering \\ Abdullah Gül University \\ Kayseri, Turkey \\ sergey.borisenok@agu.edu.tr \\ Feza Gürsey Center for Physics and Mathematics \\ Boğaziçi University \\ Istanbul, Turkey \\ borisenok@gmail.com
}

Article history:

Received 11.05.2021, Accepted 30.06.2021

\begin{abstract}
Feedback algorithms can be efficiently applied to control the basic characteristics of quantum batteries (QBs): the ergotropy, the charging power, the storage capacity and others. We invent here two alternative approaches, target repeller and speed gradient feedback, to maximize the ergotropy for bosonic types of single-qubit based quantum batteries. We demonstrate the achievability of the control goal and discuss some pros and cons of both proposed algorithms.
\end{abstract}

\section{Key words}

quantum battery, ergotropy, feedback control

\section{Introduction}

Quantum Battery (QB) is a quantum device for the efficient storage of energy and its transfer to consumption centers. Quantum batteries have different physical realizations (Dicke QB, spin QB, harmonic oscillator QB), and they vary with their basic characteristics, such as the ergotropy, the charging power, the storage capacity and others [Kamin et al., 2020].

The optimization of the working process in QBs demands the application of control methods driving the basic characteristics of the quantum battery itself and its charger. Feedback algorithms can be based on different approaches: Kolesnikov's target attractor and Fradkov's speed gradient (SG), and they both can be successfully invented for different configurations of the QB-charger system.

The basic idea to maximize an energy-based target function via speed gradient approach has been proposed in [Fradkov, 2000], and it was discussed in details in [Fradkov, 2007], including the evaluation of the excitability index for the mechanical oscillators (see Ch.4). SG control over the energy works successfully for the case of quantum systems [Borisenok, Fradkov, Proskurnikov, 2010]. Target atractor feedback has been applied to the control over the performance of qubitbased sensors [Borisenok, 2018].

Here we compare two alternative algorithms for the control over the basic working characteristics of a bosonic quantum battery. We invent them in the forms of target repeller feedback and speed gradient feedback, and then find the control field to drive the egrotropy of single-qubit QB.

\section{Mathematical Model for Bosonic Quantum Bat- tery}

We study here the particular type of QB: bosonic quantum battery based on a single qubit. The charger $A$ for such a battery $B$ is implemented via the field which controls over pumping the energy into the battery. Our model covers also the energy decay due to the coupling of the charger/QB system with the environment.

\subsection{Model Hamiltonian}

Let's consider a model quantum system consisting of two parts: the charger $A$ and the battery $B$ with the corresponding Hamiltonians $H_{A}$ and $H_{B}$. Both Hamiltonian terms have a zero ground-state energy. Apart from that there is the Hamiltonian component $H_{1}$ coupling 
the charger $A$ and the battery $B$ together [Ferraro et al., 2018]:

$$
H(t)=H_{A}+H_{B}+u(t) H_{1},
$$

where $u(t)$ is a time-dependent coupling parameter playing a role of the control signal.

The charger $A$ is described with the pair of the creation-annihilation operators $a^{+}, a$; and the bosonic harmonic oscillator battery $B$ is composed by $N$ nonmutually interacting elements marked with the index $k$, with the corresponding creation-annihilation operators $b_{k}^{+}, b_{k}$; such that the model Hamiltonian (1) is given by:

$$
\begin{aligned}
& H_{A}=\omega_{0} a^{+} a \\
& H_{B}=\omega_{0} \sum_{k} b_{k}^{+} b_{k} ; \\
& H_{1}=g \sum_{k}\left(a b_{k}^{+} a^{+} b k\right),
\end{aligned}
$$

with the positive constants $\omega_{0}$ and $g$; the Planck constant $\hbar=1$.

For simplicity we discuss here a single-qubit based quantum battery in the form of quantum oscillator. To cover the effects of the interaction between the battery and the environment, we consider also the system (2) to be placed in a Markovian bath, such that its density matrix $\rho$ is described with the Lindblad-type operator [Pechen, 2011]:

$$
\frac{d \rho}{d t}=-i\left[H_{0}+u(t) \hat{Q}, \rho\right]+\hat{L}[\rho]
$$

with

$$
\begin{aligned}
H_{0} & =\left(\omega_{0}+\frac{1}{2}\right) b^{+} b ; \\
\hat{Q} & =\frac{b^{+}+b}{\sqrt{2 \omega_{0}}} ; \\
\hat{P} & =i \sqrt{\frac{\omega_{0}}{2}}\left(b^{+}-b\right),
\end{aligned}
$$

and

$$
\begin{array}{r}
\hat{L}[\rho]=\gamma(n(t)+1)\left(2 b \rho b^{+}-\rho b^{+} b-b^{+} b \rho\right)+ \\
+\gamma n(t)\left(2 b^{+} \rho b-b b^{+} \rho-\rho b b^{+}\right) .
\end{array}
$$

The model (3)-(5) covers a decay due to the interaction of QB with the environment, and for that reason it possesses one extra control parameter $n(t)$. The positive constant $\gamma$ stands for the rate of this decay.

\subsection{Quasi-Classical Representation}

To apply the control algorithms based on the differentiable functions the quantum model (3)-(5) can be reformulated in the quasi-classical form as a set of real ordinary differential equations.

To do that we define the functions:

$$
\begin{aligned}
& E(t)=\operatorname{Tr}\left(\mathrm{H}_{0} \rho\right) ; \\
& Q(t)=\operatorname{Tr}(\hat{\mathrm{Q}} \rho) ; \\
& P(t)=\operatorname{Tr}(\hat{\mathrm{P}} \rho) .
\end{aligned}
$$

By (6) the system (3)-(5) can be re-written as [Borisenok, 2020-1]:

$$
\begin{aligned}
\dot{E}(t) & =2 \gamma\left(\omega_{0} n(t)-E(t)\right)-u(t) P(t) ; \\
\dot{Q}(t) & =P(t)-\gamma Q(t) ; \\
\dot{P}(t) & =-\omega_{0}^{2} Q(t)-\gamma P(t)-u(t) .
\end{aligned}
$$

Thus, our finalized model for the driven bosonic QB involves three ODEs for the real functions: $E(t), P(t)$ and $Q(t)$, and two control parameters: $u(t)$ and $n(t)$.

\subsection{Ergotropy}

The energy storage of quantum battery depends on the reference Hamiltonian $H$ with the finite Hilbert space of the battery system. The difference between the useful energy exacted from QB in the state $\rho$ and its energetically lowest accessible state $\sigma_{\rho}$ defines its ergotropy [Francisca et al., 2017]:

$$
W=\operatorname{Tr}(\rho \mathrm{H})-\operatorname{Tr}\left(\sigma_{\rho} \mathrm{H}\right) .
$$

In our model (7) the ergotropy could be found by (8) as:

$$
W(t)=E(t)-E_{0},
$$

where $E_{0}$ is the energy of the lowest accessible passive battery state.

\section{Repelling Feedback Control Algorithms}

There are few alternative approaches to perform an efficient feedback control over the ergotropy (9). Let's focus on two the most popular ones.

The first scheme is represented with the Kolesnikov's 'synergetic' control [Kolesnikov, 2012]. We need to define a goal function which serves to design in the dynamical system a target attractor locking the phase space trajectories in its neighborhood. That means that the trajectories converge exponentially fast to the target attractor phase space subset. The existence of such target attractor demands the permanent pumping of the energy to the dynamical system.

The alternative form is based on the family of gradient algorithms, for example, on Fradkov's speed gradient [Fradkov, 2007]. In this approach a goal function should be a differential non-negative function to drive the dynamical system toward its minimization. Fradkov's algorithm creates in the system a sort of 'target friction' which provides the maximum decay of the dynamical trajectories in the neighborhood of the control goal. As soon as the goal is achieved, Fradkov's control is off.

Very recently we invented a modification of Kolesnikov's control based on designing a target repeller in the system of small neuron population [Borisenok, 2020-2]. Here we extend our approach to the case of bosonic QB and, alternatively, propose a similar gradient algorithm based on Fradkov's control. 


\subsection{Target Repeller Feedback}

The Target Repeller Feedback (TRF) approach creates in the system (7) a dynamical target repeller driving the trajectories in the phase space far away from the certain space sub-set [Borisenok, 2020-2]. Here we apply it for the ergotropy (9) to maximize exponentially fast the function $W$ :

$$
\dot{W}(t)=\frac{W(t)}{T_{1}} .
$$

For the system (7) we use two control parameters, for that reason we need two control equations: one for the function $E(t)$ via TRF equation (10), and another for the function $P(t)$ in the form of Kolesnikov's target attractor algorithm [Kolesnikov, 2012]:

$$
\begin{aligned}
\dot{E}(t) & =\frac{E(t)-E_{0}}{T_{1}} ; \\
\dot{P}(t) & =-\frac{P(t)-P_{*}}{T_{2}} .
\end{aligned}
$$

Here $T_{1}$ and $T_{2}$ are positive constants, $P_{*}$ stands for the target stabilization $P(t)$.

Eqs (11) have the solution with the exponential behavior:

$$
\begin{aligned}
& E(t)=\left(E(0)-E_{0}\right) e^{t / T_{1}}+E_{0} \\
& \dot{P}(t)=P(0) e^{-t / T_{2}}+P_{*}\left(1-e^{-t / T_{2}}\right) .
\end{aligned}
$$

By the substitution of (11) into Eqs (7) we can restore the control signals:

$$
\begin{aligned}
n(t) & =\frac{1}{2 \gamma \omega_{0}}\left[\frac{E(t)-E_{0}}{T_{1}}+2 \gamma E(t)+u(t) P(t)\right] \\
u(t) & =\frac{P(t)-P_{*}}{T_{2}}-\gamma P(t)-\omega_{0}^{2} Q(t) .
\end{aligned}
$$

The functions (13) provide the exponential achievability of the control goal, i.e. the maximization of the ergotropy (9).

To analyze shortly the achievability of the control goal, let's study the case of a week coupling between the system and the environment: $\gamma \rightarrow 0$. Suppose that as $t \rightarrow \infty\left(t>>T_{2}\right): P(t) \rightarrow P_{*}$. Under these conditions the system (7) could be simplified. Let's assume also that the magnitude of the control field $u(t)$ is limited: $|u(t)| \leq u_{\max }$. Then we can evaluate: $Q(t) \simeq P_{*} t$ and $u(t) \simeq-\omega_{0} P_{*} t$. It applies the limit for the time:

$$
t_{\max }=\frac{u_{\max }}{\omega_{0} P_{*}} .
$$

By that we end up with the time evaluation for the ergotropy:

$$
W(t) \simeq-T_{1} P_{*} u(t)=T_{1} P_{*}^{2} \omega_{0} t,
$$

such that by (14) finally we get:

$$
W_{\max }=T_{1} P_{*} u_{\max } .
$$

Due ti its RHS Eq.(16) does nor growth infinitely.

Thus, in the frame of the given model the value of the ergotropy $W$ as a result of TRF cannot increase infinitely; its upper limit is constrained with the upper limit of the control signal $u$.

\subsection{Speed Gradient Feedback}

Now let's develop the control via the speed gradient approach based on Fradkov's feedback. To do that, we define a non-negative function of the control goal in the form:

$$
G(t)=\frac{W^{2}(t)}{2}=\frac{\left(E(t)-E_{0}\right)^{2}}{2} .
$$

This goal (17) should drive the system far away from the lowest accessible energy $E_{0}$ of $\mathrm{QB}$ to increase its ergotropy $W$.

Then, following Fradkov's approach [Fradkov, 2007], we get the control anti-gradient signals:

$$
\begin{aligned}
& n(t)=\Gamma_{n} \frac{\partial \dot{G}(t)}{\partial n} ; \\
& u(t)=\Gamma_{u} \frac{\partial \dot{G}(t)}{\partial u} .
\end{aligned}
$$

with constant positive $\Gamma_{n}, \Gamma_{u}$. Correspondingly, they become:

$$
\begin{aligned}
& n(t)=2 \gamma \omega_{0} \Gamma_{n}\left(E(t)-E_{0}\right) ; \\
& u(t)=-\Gamma_{u}\left(E(t)-E_{0}\right) P(t) .
\end{aligned}
$$

By (19) Eqs (7) could be re-written as:

$$
\begin{aligned}
\dot{W}(t) & =\left[\Gamma_{u} P^{2}(t)-2 \gamma+4 \gamma^{2} \omega_{0}^{2} \Gamma_{n}\right] W(t)-2 \gamma E_{0} \\
\dot{Q}(t) & =P(t)-\gamma Q(t) ; \\
\dot{P}(t) & =-\omega_{0}^{2} Q(t)-\gamma P(t)+\Gamma_{u}\left(E(t)-E_{0}\right) P(t),
\end{aligned}
$$

The control system (20) provides the maximization of the ergotropy (9). Particularly, we can study the achievability of the control goal (19) as $\gamma \rightarrow 0$. For $\Gamma_{u}>>\omega_{0}$ we get:

$$
\begin{gathered}
P(t) \simeq \sqrt{\frac{c_{1} e^{c_{1} t}}{\Gamma_{u}\left(c_{2}-e^{c_{1} t}\right)}} ; \\
W(t) \simeq \frac{c_{1} c_{2}}{2 \Gamma_{u}\left(c_{2}-e_{1}^{c} t\right)},
\end{gathered}
$$

with the constants based on the initial conditions:

$$
\begin{aligned}
c_{1} & =\Gamma_{u}\left[2 W(0)-P^{2}(0)\right] ; \\
c_{2} & =\frac{2 W(0)}{P(0)} .
\end{aligned}
$$


If the upper limit for the control signal magnitude is $u_{\max }$, then:

$$
|u|=\left|\Gamma_{u} W P\right| \leq u_{\max }
$$

and

$$
W_{\max }=\frac{u_{\max }}{\Gamma_{u} P_{*}} .
$$

Again, the upper limit of the control signal bounds the maximal ergotropy.

\section{Conclusions and Discussions}

The control algorithms proposed here have few distinct features:

- They are universal and do not depend on the initial conditions of the dynamical variables.

- They are robust and stable under the perturbation of the initial conditions and the relatively small external noise.

- They can be easily extended for a multi-qubit model.

The proposed approach could be applied also for different physical realizations of quantum batteries: Dicke $\mathrm{QB}$, spin $\mathrm{QB}$; and for all working stages of the quantum battery: charging, long time storage and the energy transfer to a consumption center or engine.

The construction of the repeller in the dynamical system seems to be natural in the frame of Kolesnikov's algorithm. From another hand, the definition of the dynamical attractor via the negative feedback loop could be also performed in the frame of any optimal or suboptimal approaches: Pontryagin's optimal control, Fradkov's speed gradient, and others.

The choice of the particular feedback form depends on the practical conditions. In general, the gradientbased algorithms are less energy-consuming, which is extremely important for the energy-storing quantum devices. Also such algorithms could be easily computed in the real time regime. From another side, the gradientbased approaches are less accurate in the achievement of the goal to compare with target attractor / repeller feedback. Thus, the basic criteria for the choice should be: the computational time cost and the cost of the energy that we need to pump into the system to support the control dynamics. Pros and cons of different alternative approaches will be a matter of our following research.

\section{Acknowledgements}

We thank Prof. Dr. A. Fradkov for valuable productive discussion on the speed gradient adaptation to the ergotropy control.

\section{References}

Borisenok, S. (2018). Control over performance of qubit-based sensors. Cybernetics and Physics, 7(3), pp. 93-95.

Borisenok, S. (2020). Ergotropy of quantum battery controlled via target attractor feedback. IOSR Journal of Applied Physics, 12(3), pp. 43-47.

Borisenok, S. (2020). Suppressing epileptiform dynamics in small Hodgkin-Huxley neuron clusters via target repeller-attractor feedback. IOSR Journal of Mathematics, 16(4), pp. 41-47.

Borisenok, S., Fradkov, A., and Proskurnikov, A. (2010). Speed gradient control of qubit state. Periodic Control Systems, 4(1), pp. 81-86.

Ferraro, D., Campisi, M., Andolina, G., Pellegrini, V., and Polini, M. (2018). High-power collective charging of a solid-state quantum battery. Physical Review Letters, 120(11), p. 117702.

Fradkov, A. (2000). A nonlinear philosophy for nonlinear systems. Proceedings of the 39th IEEE Conference on Decision and Control (Cat. No.00CH37187), pp. 4397-4402.

Fradkov, A. (2007). Cybernetical Physics: From Control of Chaos to Quantum Control, Springer, Berlin.

Francica, G., Goold, J., Plastina, F., and Paternostro, M. (2017). Daemonic ergotropy: enhanced work extraction from quantum correlations. npj Quantum Inf., 3, p. 12.

Kamin, F. H., Tabesh, F. T., Salimi, S., Kheirandish, F., and Santos, A. C. (2020). Non-Markovian effects on charging and self-discharging processes of quantum batteries. New Journal of Physics, 22, p. 083007.

Kolesnikov, A. (2012). Synergetic control methods of complex systems, URSS Publ., Moscow.

Pechen, A. (2011) Engineering arbitrary pure and mixed quantum states. Physical Review A, 84, p. 042106. 